\title{
PRODUCTOS AGROALIMENTARIOS DE CALIDAD Y TURISMO EN ESPAÑA: ESTRATEGIAS PARA EL DESARROLLO LOCAL*
}

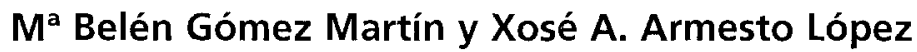 \\ Dpt. de Geografia Física i Anàlisi Geogràfica Regional, Facultat de Geografia i \\ Història, Universitat de Barcelona. C/ Baldiri Reixac s/n, Barcelona 08028 \\ bgomez@ub.edu armesto@ub.edu
}

\begin{abstract}
Resumen: Este artículo muestra las relaciones existentes entre el turismo y las producciones agrarias basadas en productos tradicionales, revalorizados en los últimos tiempos gracias a las denominaciones de calidad. Los productos agroalimentarios se configuran, en este contexto, como recursos turísticos gastronómicos que posibilitan el desarrollo del turismo y, a la vez, el turismo se convierte en herramienta para la promoción y comercialización de los productos agroalimentarios de calidad. Ambas circunstancias se aúnan con un objetivo común: el desarrollo local de territorios tradicionalmente considerados marginales en el ámbito regional.
\end{abstract}

Palabras clave: gastronomía, turismo, alimentos de calidad, desarrollo local, agricultura, España.

\begin{abstract}
This article shows the relations between the tourism, and the features of an agrarian production based in traditional products, revalued in the last times due to the guarantees of origin. The food and agriculture products take shape, in this context, as a gastronomic tourists resources that make possible the development of tourism and, at the same time, the tourism turns into a promotional and marketing tool of the quality food and agriculture products. Both circumstances join forces with a common objective: the local development of territories traditionally considered as marginal in the regional context.
\end{abstract}

Key words: gastronomy, tourism, quality food, local development, agriculture, Spain.

\footnotetext{
* Recibido: 16-03-04. Aceptado: 04-11-04.
} 


\section{Introducción}

En los últimos años, el desarrollo del turismo en los diferentes espacios o territorios exhibe características singulares que se interpretan como síntomas de un cambio en el sistema y que evidencian el inicio de una nueva etapa en la historia de este importante fenómeno económico y social. Esta nueva fase, que se inicia a finales de los años ochenta y que se conoce con el nombre de postfordista, configura un escenario original en el que aparecen nuevos procesos de producción y consumo turístico en los que el espacio geográfico adquiere un renovado valor y una dimensión diferente (López Palomeque, 1999).

Las nuevas tendencias que se observan en la producción y consumo de turismo cultural no pueden desvincularse del nuevo paradigma turístico (Urry, $1990 \mathrm{y}$ Nuryanti, 1996, citado en Antón, 2000). En este sentido, la singularización del espacio turístico a través de la valoración productiva y simbólica de aquellos elementos del patrimonio cultural que le confieren identidad territorial o el renovado gusto por la cultura en todas sus manifestaciones o la contemplación de los aspectos culturales descle una vertiente más recreativa y lúdica, son rasgos que evidencian las nuevas formas de explotación turística (Antón, 2000). De hecho, el protagonismo que en los últimos años adquieren algunas formas de turismo cultural hasta ahora consideradas complementarias, como por ejemplo el turismo gastronómico, sólo puede entenderse dentro del marco de las renovadas tendencias.

En el turismo gastronómico, los productos alimenticios y las especialidades culinarias se convierten en vehículo de acercamiento cultural en la medida en que se configuran como elementos identificadores de pueblos y territorios. Esta nueva forma de aproximación a la cultura de un lugar, región o país tiene un carácter más activo y vivencial, lúdico y festivo y, a priori, procura un desarrollo sostenible que pasa por: a) asegurar el mantenimiento de los recursos turísticos; b) mejorar los niveles de bienestar de la población que se encarga de su producción y comercialización y, por último, c) garantizar la satisfacción de la experiencia turística por la vía de la calidad. Esta satisfacción de la experiencia turística por la vía de la calidad va ligada con frecuencia a la existencia y promoción de productos alimentarios con marchamo de calidad.

La calidad de los alimentos es uno de los pilares de la nueva orientación en lá Política Agraria Comunitaria (PAC). Esta nueva perspectiva es consecuencia directa de la situación que se había generado después de la II Guerra Mundial, en la que una Europa arrasada por la conflagración tenía que recuperarse económicamente a marchas forzadas incrementando todas las vertientes productivas, entre ellas, por supuesto, la agraria. Esta situación hizo que se acumulasen gran cantidad de excedentes, que la población rural continuara disminuyendo y desestructurándose socialmente y que el medio ambiente se deteriorase con rapidez, así que en la década de 
los ochenta los dirigentes comunitarios comenzaron a elaborar discursos y primeras propuestas para intentar paliar estos desajustes?

Actualmente, el campo español se encuentra condicionado por la política europea que trata nominalmente de basarse en tres premisas: el mantenimiento de la función productiva, la consideración del mundo rural como base social de Europa y la protección y mejora medioambiental. Es en el primer objetivo donde se debe incluir la calidad que aparece como eje fundamental de la nueva agricultura europea y que pretende sustituir la búsqueda del aumento de la producción. "La noción de calidad es, para la década en que vivimos, lo que la noción de cantidad fue para los años de posguerra" (Hervieu, 1996: 117). Esta nueva situación en la que se pretende contemplar al medio rural en su conjunto y no exclusivamente bajo el prisma de la producción agraria hace que algunos autores hablen de que el campo europeo está en plena transición postproductivista. A este campo actual se le exigen nuevas ofertas que combinan la tradición y la innovación; así, encontramos cada vez más que el medio rural ha de servir para proveer a los mercados urbanos además de alimentos, de otros bienes inmateriales como naturaleza, paisaje, equilibrio territorial, cultura, salud, gastronomía o turismo, todos ellos contemplados desde el propio consumidor y basados en el marco conceptual del postfordismo y el postmodernismo (Rubio, 1999). En el marco de esta filosofía es donde debemos incluir la idea general del Desarrollo rural sostenible, donde los alimentos de calidad se revelan como un instrumento más para poder alcanzarlo.

En definitiva, estas necesidades de los consumidores en referencia al espacio rural y centradas en la búsqueda de la calidad en su dimensión más amplia, hacen que la localidad y la región resurjan con fuerza en un mundo que nos empeñamos en definir como globalizado. Las denominaciones de origen para productos agrarios, en sus diversas formas de protección, consiguen fortalecer la relación geográfica entre las producciones, el territorio, los productores, los elaboradores y los consumidores. Por tanto, parece evidente que la imbricación entre este tipo de producciones catalogadas y el consumidor no se realiza solo en el supermercado sino que además existe un vínculo que se establece en función de la movilidad ligada al ocio de la población urbana que encuentra un aliciente más en la gastronomía y los productos típicos del lugar elegido.

\section{Turismo cultural y gastronomía en España}

Las transformaciones socioeconómicas experimentadas en las dos últimas décadas han afectado a la delimitación del concepto de patrimonio cultural. Esta noción se ha ampliado, incorporando a los monumentos, los grupos de edificios y los lugares his- 
tóricos (UNESCO, 1972), nuevos elementos vinculados a la llamada cultura tradicional y popular. De este modo, el patrimonio cultural se entiende hoy como "el conjunto de elementos naturales y culturales, tangibles e intangibles, que son heredados o creados recientemente y mediante los cuales, grupos sociales reconocen su identidad y se comprometen a transmitirla a generaciones futuras de una manera mejor y enriquecida" (UNESCO, 1998).

El concepto de turismo cultural ha evolucionado de forma paralela al de patrimonio cultural, siendo entendido hoy como el movimiento temporal de personas hacia una atracción cultural (sitios patrimoniales, arte, folklore, eventos populares, acontecimientos programados, etc.) fuera de su lugar habitual de residencia, con la intención de conocer, comprender y experimentar para cultivarse y satisfacer sus necesidades turísticas (Grande, 2001; Montero, Gutiérrez y Díaz, 2001; Antón, 2000). La gastronomía, entendida como el valor cultural que se concede a los alimentos o a la forma de prepararlos, por su identificación con un territorio o grupo social es uno de los elementos incorporados con fuerza al nuevo concepto de patrimonio cultural y uno de los recursos turísticos sobrevalorado en los últimos años, por su capacidad de satisfacer las nuevas exigencias de consumo de la demanda de turismo cultural.

La gastronomía, como recurso turístico, no sólo es apreciada por su propio valor intrínseco sino también por su carácter simbólico, en la medida en que actúa como identificador de pueblos y territorios. En este sentido, se debe considerar que la identidad adquiere una importancia destacada en una sociedad globalizada, donde la propia cultura ha sufrido un proceso de estandarización (Grande, 2001). Esta homogeneización, que en gastronomía podría ser identificada con el proceso de MacDonalización y Coca-Colalización o con el imperialismo culinario norteamericano (extendido bajo el anterior paradigma fordista), ha generado como respuesta un deseo por lo auténtico y tradicional (postfordismo) (Ritzer, 1998; Moulin, 2000). Este deseo se ha materializado, por un lado, en el crecimiento de una restauración ligada a la cocina popular y a los productos de calidad autóctonos y, por otro, en la consolidación de una nueva submodalidad de turismo: el turismo gastronómico.

La gastronomía, como recurso turístico, también es apreciada por su adecuación a las nuevas tendencias de consumo cultural. La gastronomía permite aproximarse a la cultura de un modo más vivencial y participativo (postmodernismo), no estrictamente contemplativo. Además, bajo determinadas formas de explotación, puede generar productos turísticos de alto valor añadido $y$, en ocasiones, con carácter de exclusividad (post-turismo).

Todos estos aspectos, de interés en el nuevo sistema de producción y consumo turístico, hacen que la gastronomía se convierta con frecuencia en factor de atracción y en elemento integrante de la imagen del destino turístico, jugando así un importante papel en el proceso de decisión de compra que realiza el turista-consumidor 
(Chon, 1990). De hecho, según una investigación de Gallarza, Gil y Calderón (2002) realizada sobre un total de 25 estudios de TDI (tourism destination image o imagen del destino turístico), la gastronomía es uno de los atributos que más importancia tiene en la formación de imágenes del destino turístico por parte de la demanda (ocupaba la quinta posición en un ranking integrado por 20 variables) y, a la vez, uno de los que más influencia ejerce en los niveles de satisfacción que experimenta el visitante.

En el panorama turístico español, el turismo gastronómico es uno de los productos emergentes que más se está desarrollando en los últimos años. El elevado volumen de productos turísticos que tienen como base fundamental los recursos gastronómicos (en este sentido, destaca el aumento notable en el número de rutas turisticas gastronómicas) y el incremento constante en la cifra de establecimientos de restauración ${ }^{2}$ dan buena prueba de ello. El potencial de recursos que ofrece el país sirve de base a este creciente desarrollo: la diversidad geográfica y cultural proporciona una gran variedad de alimentos y de formas de prepararlos y, en consecuencia, configura y da contenido a los conceptos de cocina local (cocina pirenaica, cocina malagueña, cocina empordanesa, de la Maragatería..), cocina regional (cocina andaluza, gallega, vasca, catalana...), cocina de grandes espacios geográficos (cocina mediterránea, cocina cantábrica, de interior....), etc.

Los aspectos gastronómicos se configuran en la actualidad española como recursos turísticos complementarios o como recursos básicos que participan en la elaboración de productos turísticos. En el primer caso, los recursos gastronómicos no provocan por sí mismos desplazamientos turísticos pero generan un valor añadido a la imagen del destino, crean un efecto diferenciador frente a los competidores y juegan un papel trascendental en la evaluación del grado de satisfacción del visitante. La restauración, entendida como el conjunto de establecimientos especializados en la oferta de platos tradicionales de la cocina regional o local, es la herramienta que permite la aproximación a los recursos gastronómicos de un lugar, por lo que de su número y calidad depende la valoración que realiza el turista del destino. No en vano se puede decir que en España, el crecimiento en el inventario de restaurantes ha sido impulsado, muy especialmente, por la actividad turística y el deseo de explotar ese recurso complementario. De hecho, son las CC.AA. más turísticas las que presentan un mayor número en este tipo de establecimientos y las que mayores porcentajes de crecimiento han experimentado. Los recursos gastronómicos y las herramientas utilizadas para su puesta en uso ayudan a configurar una imagen de calidad del destino que se pone de manifiesto en los diferentes materiales divulgativos de carácter promocional: la información icónica y verbal de los folletos turísticos españoles concede una gran importancia a los aspectos y a la oferta gastronómica como parte integrante fundamental de la experiencia turística. No en vano, la información referente a los recursos y/o la oferta gastronómica o bien, posee un espacio diferenciado en los folletos generales y/o bien, aparece bajo un folleto específico de carácter gastronómico. 
En el segundo caso, los recursos gastronómicos se convierten en protagonistas, jugando un papel decisivo en el desplazamiento de turistas hacia ciertas áreas geográficas. Los alimentos y/o la forma de prepararlos son los elementos fundamentales del producto turístico, por lo que la restauración, la existencia de pequeño comercio especializado en productos tradicionales y la presencia de museos temáticos de pequeña y mediana dimensión adquieren una importancia fundamental en la experiencia turística. De hecho, los turistas de esta modalidad realizan su principal gasto en el rubro alimentación que, en este caso, no sólo incluye el consumo local sino también la adquisición de productos alimenticios como souvenirs o para consumo propio en los lugares de origen.

La tematización de los productos turísticos en torno a la gastronomía se configura como una estrategia más de diversificación de la oferta y adaptación a los nuevos gustos que manifiesta la demanda. La incorporación de estos recursos básicos en el mercado español adopta tres formas diferenciadas:

1. Rutas temáticas. Se aplican a diferentes escalas y consisten en itinerarios que tienen como objetivo dar a conocer un determinado territorio a través de visitas de corta duración que giran en torno a un mismo aspecto o tema gastronómico (los alimentos y la forma de prepararlos se convierten en elementos vertebradores). En ocasiones la ruta gira en torno a un producto (aceite, queso, vino, jamón); otras veces gira en torno a un plato específico (asados, gazpachos, paellas, guisos, mariscadas). Las rutas gastronómicas ofrecen una serie de placeres y actividades relacionadas con los elementos distintivos de la misma: degustación de productos o platos, visitas guiadas para conocer el proceso de producción agroindustrial del recurso (en ocasiones en vivo, en ocasiones se reproduce en museos), compras en establecimientos especializados, visitas a mercados tradicionales, etc. Hay que tener en cuenta que las rutas permiten integrar en un producto, elementos que, individualmente sólo podrían configurarse como recursos complementarios de otras modalidades turísticas, pero que unidos vertebran y generan un producto final de gran valor. Si bien este tipo de itinerarios se inició como una forma de turismo individual que organizaba por su cuenta los pormenores del viaje (dejando a su elección la utilización de los diferentes servicios turísticos), en la actualidad se encuentra también muy ligado a los viajes colectivos organizados y, en especial, al turismo social para la tercera edad.

2. Viaje al destino. En estos casos la demanda se dirige hacia destinos de reconocido prestigio gastronómico y con una importante oferta de restauración, bajo el concepto de viaje organizado o no. Suelen ser lugares que utilizan los productos gastronómicos como principales factores de atracción aunque conjuntamente también se incluyen otros atractivos culturales o naturales de reconocido valor. En ocasiones estos viajes se producen a lo largo de todo el año 
(sirva como ejemplo, el caso de Segovia con el cochinillo); en cambio en otras presentan un marcado carácter estacional ligado a las particulariclades de la materia prima (podemos citar como ejemplo los viajes otoñales que se producen con motivo de la degustación de setas en el Pirineo catalán).

3. Eventos. Incluyen celebraciones tematizadas (ferias tradicionales, mercados y fiestas de exaltación de productos o costumbres típicas), que trascienden el ámbito estrictamente local, y que generan importantes desplazamientos de visitantes. El caso gallego es muy significativo en cuanto a la organización de este tipo de acontecimientos: citamos como ejemplo las festividades en torno al mejillón en Corcubión, Lorbé, A Coruña o Combarro. Muchas de estas celebraciones presentan la Declaración de Interés Turístico, una distinción que se otorga a aquellas fiestas o acontecimientos que suponen una manifestación de los valores culturales y de tradición popular, con especial consideración a sus características etnológicas, y que tienen una especial importancia como atractivo turístico. De nuevo, el caso gallego vuelve a evidenciar la importancia de estos recursos en su sistema turistico: de las 22 Fiestas Declaradas de Interés Turístico Nacional en Galicia 5 tienen carácter gastronómico. Del mismo modo, de las 35 Fiestas de Galicia de Interés Turístico (distinción de carácter regional) 16 giran en torno a productos $y / o$ platos típicos de la tierra.

Las tres formas de comercialización presentadas muestran elementos comunes relativos a la tendencia que este tipo de productos tienen hacia una animación y teatralización de los recursos y de los espacios (para permitir el acercamiento y el disfrute de los mismos) y hacia la interpretación participativa (para permitir experiencias creativas y enriquecedoras tan demandadas hoy por los nuevos turistas).

Cuando estos recursos se convierten en protagonistas, la imagen turística del país, región o destino se asocia a los aspectos gastronómicos, remitiendo a ellos de forma directa los eslóganes y logotipos turísticos. Sirva como ejemplo el caso de Asturias, con eslogan de campaña (o marca genérica de Principado) Asturias, paraíso natural y eslogan de producto (o submarca) Saboreando Asturias.

Este papel relevante de los aspectos gastronómicos se advierte muy bien en los stands promocionales que algunas CC.AA., provincias o destinos presentan en los salones y ferias de turismo (Fitur en Madrid, ITB en Berlín, SITC en Barcelona, etc.), donde exhiben y permiten la degustación de los principales productos y especialidades culinarias. También se deja ver en la elevada existencia de folletos específicos, exposiciones-muestras itinerantes (sirva como ejemplo la organizada en el 2003 por la Diputación de Córdoba y denominada Córdoba: el sabor de una cultura) y guías turísticas especializadas en gastronomía (Buen Viaje: las mejores rutas gastronómicas por España...). 
Este papel de los aspectos gastronómicos en el desarrollo turístico se manifiesta también en algunas de las iniciativas llevadas a cabo en España en los últimos años:

a) Creación de Etiquetas de Calidad para realzar productos alimentarios de rango tradicional elaborados en torno a una ruta o región turística consolidada. El caso más conocido es el de la creación de la etiqueta de calidad Tradición del Camino para realzar los productos alimentarios tradicionales elaborados en el entorno del legendario Camino de Santiago. Seiscientas treinta y cinco empresas artesanas, pertenecientes a tres países, Francia, Portugal y España, agrupadas en dieciséis asociaciones que corresponden a once regiones bien diferenciadas, lanzan al mercado europeo esta marca. Entre sus objetivos figuran favorecer las tradiciones gastronómicas regionales como parte de la cultura del Camino de Santiago, abrir nuevas vías de promoción y distribución en mercados turísticos y culturales, así como generar nuevos puestos de trabajo, en particular en zonas rurales y de montaña aisladas de los circuitos económicos habituales.

b) Fundación de asociaciones público-privadas especializadas para dinamizar turisticamente el territorio a través de la gastronomia. Así, por ejemplo, Turisme de Catalunya crea en el año 2002 el llamado Club de Gastronomia con el objetivo de promocionar el país mediante la gastronomía. En él participan diversos colectivos de cocineros y restauradores del ámbito local o comarcal, organizaciones como el Institut Català de la Cuina, productores, profesionales de la cultura y las ciencias, así como diversos agentes turísticos. Una de las principales aportaciones del Chub de Gastronomia ha sido la elaboración de una Agenda Gastronómica de Catalunya.

c) Firma de convenios para impulsar un turismo de calidad ligado a la gastronomía. La Secretaría de Estado de Comercio y Turismo, la Federación Española de Hostelería, la Fundación Española de Nutrición y la Academia Española de Gastronomía, han firmado un convenio para dirigir el turismo de calidad hacia España. Uno de los medios más apropiados, y que se perfila como elemento sustancial para conseguir este objetivo, es el de promocionar en el extranjero la gastronomía española entendida como expresión de cultura. El proyecto implica la programación y puesta en práctica de acciones que establezcan estándares e instrumentos que reconozcan e impulsen la calidad gastronómica española en los establecimientos radicados en el extranjero. Así, como primer plan de acción, se presenta el Proyecto de Excelencia Gastronómica. Su finalidad es distinguir los restaurantes españoles en el extranjero que alcancen un determinado nivel de calidad y de "españolidad". A este Convenio podrán adherirse otras empresas y entidades del mundo del turismo y la alimentación que apoyen esta iniciativa, contribuyendo al beneficio común del sector. 


\section{Los nexos entre la calidad y el territorio}

El turismo gastronómico fundamenta su desarrollo en la presencia de unos productos y una cocina de cualidades especiales. La identificación de esos productos y esa cocina diferenciada y singular va unida con frecuencia a la tradición y a la existencia de alimentos y establecimientos con distintivos de calidad.

\subsection{Apuntes sobre el concepto de calidad agroalimentaria}

En la actualidad este concepto es uno de los sujetos más estudiados en las investigaciones agrarias y rurales. Para entender su significado real hay que remontarse al principio de la actividad agraria. En inicio, los agricultores tuvieron una preocupación básica: incrementar los rendimientos de sus producciones para poder acumular excedentes que permitieran su supervivencia en momentos de crisis productivas o en estaciones desfavorables para el cultivo. Este paradigma ha continuado hasta épocas bien recientes y todavía hoy continúa presente de un modo u otro en la mayoría de los agricultores que utilizan los métodos productivistas como forma de gestión de sus explotaciones y que persiguen, no la acumulación de excedentes para afrontar posibles crisis, sino la acumulación de capital para engrosar sus cuentas personales. Deseo natural y muy lícito que aparece en cualquier otra actividad productiva.

En la situación anteriormente descrita el concepto de calidad fue borrado de un plumazo, de este modo las producciones del sector primario adquirieron características propias del sector secundario. Esta tendencia se concretó especialmente en los llamados países del primer mundo a partir del fin de la Ir Guerra Mundial.

La calidad se puede definir como la superioridad en su categoria, es decir, un estadio que define la condición óptima de un producto alimenticio. La calidad interna de un producto lleva implícitas las siguientes formas (Dedeire, 2001): a) la calidad higiénica, referida a la ausencia de toxicidad química; b) la calidad nutricional, relacionada con el equilibrio dietético; c) la calidad organoléptica, que se define por las sensaciones gustativas, olfativas, táctiles o visuales; d) la calidad de uso, relativa a los aspectos relacionados con la conservación y facilidad de uso. Para B. Ilbery y $\mathrm{M}$. Kneafsey (2000) la calidad agroalimentaria es abordada desde tres perspectivas bien diferenciadas: a) la de las instituciones, los parámetros que definen la calidad en este caso están muy relacionados con el concepto de higiene; b) la de los productores, que centran la definición del término en la materia prima y en los métodos de elaboración; c) la de los consumidores, que basan su significado fundamentalmente en su experiencia adquirida a lo largo de los años y que introducen la dimensión de la confianza y el riesgo. 
La eclosión de la calidad como objetivo a conseguir por los consumidores, las instituciones y los productores coincide con el momento en el que surgen a la luz los escándalos alimentarios más importantes. De ahí, que este sea la vertiente más tratada por los investigadores rurales europeos en estos últimos tiempos. En España, el desastre del aceite de colza adulterado que provocó en la década de los ochenta numerosas víctimas y dejó secuelas permanentes a centenares de personas; las frecuentes intoxicaciones, sobre todo en épocas estivales, por la bacteria salmonela; y en los últimos años fundamentalmente la Encefalopatía Espongiforme Bovina (EEB) o mal de las vacas locas, han puesto en guardia a buena parte de los consumidores (Armesto, 2003).

\subsection{Los alimentos de calidad en España: el territorio como primera referencia}

Los productos de calidad se pueden definir como aquellos que se encuentran vinculados a un territorio de referencia y que además, desde el punto de vista cultural, respetan unas tradiciones arraigadas en el tiempo en lo relativo a sus modos de obtención o elaboración. Estas circunstancias acaban por conferirles unas características cualitativas propias que los han de diferenciar de posibles competidores.

Desde la Unión Europea (UE) se ha tomado conciencia de la riqueza cultural y de las oportunidades de desarrollo que ofrecen los modos de producción que recuperan aspectos tradicionales y que preservan en mayor medida el medio ambiente. Para fomentarlos y protegerlos en el año 1992 surgieron dos reglamentos asociados, el 2081/92 y el 2082/92; en ellos se definían las tres figuras esenciales de valorización agraria $^{3}$ : las Denominaciones de Origen Protegidas (DOP), las Indicaciones Geográficas Protegidas (IGP) y las Especialidades Tradicionales Garantizadas (ETG). Además, en 1999 se aprobó el Reglamento (CE) n 1493/99 que contiene la regulación y sistema de protección de los vinos de calidad producidos en regiones determinadas (vcprd).

Las dos primeras, junto con la referente a la producción vitivinícola son las que tienen un componente territorial más claro tal como se demuestra en sus definiciones.

a) Denominación de Origen Protegida: el nombre de una región, de un lugar determinado o, en casos excepcionales de un país, que sirve para designar un producto agrícola o un producto alimenticio originario de dicha región, de dicho lugar determinado o de dicho país, y cuya calidad o características se deban fundamental o exclusivamente al medio geográfico con sus factores naturales y humanos, y cuya producción, transformación y elaboración se realicen en la zona geográfica delimitada (Reglamento CE, $N^{\circ} 2081 / 92$ ). 
b) Indicaciones Geográficas Protegidas: el nombre de una región, de un lugar determinado o, en casos excepcionales, de un país, que sirve para designar un producto agrícola o un producto alimenticio originario de dicha región, de dicho lugar determinado o de dicho país y que posea una cualidad determinada, una reputación u otra característica que pueda atribuirse a dicho origen geográfico, y cuya producción y/o transformación y/o elaboración se realicen en la zona geográfica determinada (Reglamento CE, $N^{\circ} 2081 / 92$ ).

c) Especialidades Tradicionales Garantizadas: esta es la figura en la que el territorio aparece en una posición más marginal, de hecho en su definición ni aparece como tal. También hay que decir que además es la que tiene una representatividad más limitada, escapando al sentido postproductivista que sí tienen las anteriores. Se define como la denominación que sin base geográfica determinada protege elaboraciones tradicionales de productos agrícolas y alimenticios con características específicas, que se distinguen de otros de su mismo tipo (Reglamento CE, No 2082/92).

d) Vinos de calidad producidos en regiones determinadas: las cuatro categorías que forman esta figura (vinos de licor de calidad, vinos espumosos de calidad, vinos de aguja de calidad y vinos de calidad restantes), deben estar producidos en regiones delimitadas con precisión por parte de los respectivos Estados $^{4}$. El nombre geográfico que designe una región determinada debe ser suficientemente preciso y estar notoriamente ligado al área de producción de manera que se eviten las confusiones. Estos vinos solo podrán elaborarse a partir de las variedades que aparecen en este reglamento y su transformación debe efectuarse en esa misma región en la que se haya recolectado la uva (Reglamento CE, $N^{\circ}$ 1493/99).

Esta nueva reglamentación dimanada descle la UE hizo que en España las diversas Administraciones tuvieran que adaptar sus instrumentos y así en 1994 desde el Ministerio de Agricultura, Pesca y Alimentación se equipararon las antiguas Denominaciones de Origen y Denominaciones Específicas con las DOP e IGP. Además en el año 2003 apareció la nueva Ley de la viña y el vino que reformaba definitivamente la anterior que databa del año 1970.

En España, en los últimos diez años, ha proliferado el surgimiento de identificadores de calidad para los alimentos, promovidos especialmente desde las Comunidades Autónomas teniendo en ocasiones su reflejo en la citada Normativa Comunitaria. Sirva como referencia el dato relativo al número de Denominaciones de Origen: para el año 1987, solo doce, para 1992, veintiséis, en 1997 el número había crecido hasta las 49, mientras que en el año 2002 la cantidad de DOP e IGP había llegado a 88. A comienzos de 2004 hay en España 102 productos amparados bajo estas dos figuras fundamentales (Véase Tabla 1), siendo uno de los Estados con más figu- 
ras de este tipo dentro del contexto de la Unión. Independientemente de los alimentos reconocidos como de calidad por la UE, existen toda una serie de alimentos paralelos que en el mejor de los casos cuentan con una figura de garantía regional emitida por la Comunidad Autónoma (p. ej. morcilla de Aragón, Queso del Cebreiro, jamón de Trévelez, Anchoas de la Escala ...), o que no cuentan con ningún marchamo de calidad oficial pero que, sin embargo, tienen una notable aceptación y prestigio entre los consumidores (marisco gallego, embutidos del Pirineo catalán, aceite de oliva de numerosas áreas andaluzas exentas de certificación de origen, productos de chacina de numerosas comarcas castellanas, etc.). No se pueden pasar por alto las etiquetas de calidad aplicadas a productos alimentarios elaborados alrededor de una ruta o región turística consolidad (véase lo explicado en el apartado 2 de este artículo en relación a la marca Tradición del Camino).

Tabla 1. Número de DOP e IGP según la clasificación del MAPA (Enero de 2004).

\begin{tabular}{|lc|}
\hline Tipo de producción & $\mathbf{N}^{\mathbf{0}}$ Productos protegidos \\
\hline Aceites de oliva virgen & 19 \\
Arroces & 3 \\
Carnes frescas & 14 \\
Condimentos y especias & 2 \\
Embutidos y otros productos cárnicos & 5 \\
Frutas & 12 \\
Hortalizas & 10 \\
Jamones & 4 \\
Legumbres & 4 \\
Mieles & 3 \\
Quesos y productos lácteos & 20 \\
Turrón, mazapán y dulces & 2 \\
Varios & 4 \\
Total & 102 \\
\hline
\end{tabular}

Fuente: www.mapya.es/alimentacion/pags/Denominacion/resultado.asp MAPA (2004)

Una cuestión que aparece de manera clara en el ideario del consumidor es la identificación de los alimentos amparados por alguna de las figuras de protección con lo que C. Sage (2003) denomina "Alimentos buenos". El consumidor interpreta el origen como una señal que le permite atribuir un valor cualitativo a un bien, una calidad limpia y exenta de riesgos. Esos productos más buenos o sanos están directamente relacionados con espacios concretos, regiones, localidades o lugares, donde se cultivan, se alimentan o elaboran estas producciones. En el caso español, las referencias escalares más comunes en las figuras de protección agroalimentaria son las locales (Gómez Martín y Armesto López, 2002). Esa misma idea de calidad asociada al retorno a la localidad aparece como uno de los argumentos centrales en un reciente trabajo de M. Winter (2003). 
De cualquier manera, los distintos tipos de denominaciones de calidad no solo deben ser contemplados desde la escala de la localidad, también deben ser contextualizados en lo global; el papel en las negociaciones del General Agreement on Tariffs and Trade (GATT), las regulaciones que se imponen desde la Organización Mundial del Comercio (OMC), o la apuesta decidida de la UE, son solo algunos ejemplos de la dimensión global que alcanzan este tipo de producciones. Precisamente es en este contexto en el que llevan apareciendo hace ya tiempo situaciones en las que se pone en entredicho la actitud al respecto de estos productos de algunos países de fuera de la Unión, fundamentalmente los del grupo de Cairns, que utilizan algunas denominaciones de manera fraudulenta según la UE, amparándose en el argumento de que sus poblaciones son mayoritariamente de procedencia europea y que, por tanto, su cultura, y por ende, su gastronomía también, generándose, de este modo, situaciones en las que las antiguas metrópolis europeas acusan de competencia desleal a estos países ${ }^{5}$.

En cuanto a la distribución territorial en España de estos productos, las CC.AA. en las que más abundan son (Véase Tabla 2): Andalucía, Cataluña y Castilla y León. Existen diversos motivos por los que aparece un desequilibrio tan notable entre estas tres regiones y las que tienen menos figuras de protección geográfica, Madrid, Cantabria y Asturias. Tanto Castilla y León como Andalucía son regiones de vasta extensión y gran variedad de paisajes agrarios (sobre todo esta última), cosa que provoca que también haya como resultado una nómina importante de productos diferenciados. A estos condicionantes de marcado carácter geográfico, hay que añadirles una cierta perificidad económica de los territorios rurales de las dos comunidades, cuestión que ha contribuido de manera decisiva en el mantenimiento de formatos productivos ya en desuso en otros lugares. El caso catalán no comparte esas características ya que se trata de un territorio de tamaño medio, aunque eso sí, muy variado geográficamente con lo que ello supone en las producciones agroalimentarias, cuenta. con un importante mercado interno y una incuestionable proyección comercial exterior, con unos sectores agrarios y alimentarios muy modernizados y tecnificados, una importante red de iniciativas rurales y una población activa agraria mucho menor que en los otros casos. Esas regiones que tienen menos representatividad en esta cuestión tienen en común su extensión limitada (ninguna de ellas sobrepasa los $11.000 \mathrm{~km}^{2}$ ). En el contexto de las producciones agrarias diferenciadas, el caso de Madrid es un tanto especial, ya que la tasa de urbanización es superior a cualquier otra región, siendo además el porcentaje de activos agrarios realmente residual $(0,3 \%)$; por tanto, parece lógico que sea una de las CC.AA. con una menor proporción de Denominaciones de Origen declaradas. Los otros dos ámbitos señalados, Asturias y Cantabria, son dos regiones relativamente homogéneas desde el punto de vista físico al quedar constreñidas en una latitud similar entre las altas cumbres de la Cordillera Cantábrica y el Mar del mismo nombre, y ser limítrofes entre sí. Esta relativa homogeneidad, sobre todo si se compara con otros ámbitos autonómicos de España, no basta por sí sola para explicar esta menor representatividad de las denominaciones 
geográficas de calidad; un factor determinante lo juegan los propios gobiernos regionales y sus decisiones al respecto de la promoción de sus productos típicos. El caso asturiano es más que paradigmático, ya que a pesar de solo tener reconocidas 2 DOP y 2 IGP, tiene reconocidas y amparadas bajo una figura de carácter regional un buen número de producciones (contabilizando solo los quesos el número llega hasta las 24).

Tabla 2. Distribución de las principales Denominaciones de Origen en España (2004).

\begin{tabular}{|l|c|c|c|c|}
\hline & DOP & IGP & vcprd & TOTAL \\
\hline Andalucía & 13 & 2 & 4 & 34 \\
Aragón & 3 & 3 & 5 & 17 \\
Canarias & 2 & 0 & 8 & 11 \\
Cantabria & 3 & 1 & 0 & 4 \\
Castilla y León & 3 & 10 & 5 & 26 \\
Castilla - La Mancha & 6 & 3 & 7 & 21 \\
Cataluña & 7 & 9 & 12 & 29 \\
Comuniciad Valenciana & 4 & 5 & 4 & 16 \\
Extremadura & 9 & 2 & 2 & 14 \\
Galicia & 2 & 4 & 5 & 14 \\
Islas Baleares & 2 & 1 & 2 & 11 \\
La Rioja & 0 & 2 & 2 & 9 \\
Madricl & 0 & 2 & 1 & 4 \\
Navarra & 3 & 4 & 3 & 11 \\
País Vasco & 1 & 0 & 5 & 6 \\
Principado de Asturias & 2 & 2 & 0 & 5 \\
Región de Murcia & 4 & 0 & 3 & 10 \\
TOTAL & 64 & 50 & 68 & 242 \\
\hline
\end{tabular}

Fuente: www.mapya.es/alimentacion/pags/Denominacion/resultado.asp MAPA (2004)

Entre las DOP son Andalucía y Extremadura las dos Comunidades en las que más abunda este tipo de figura; el hecho de que sean dos regiones especialmente grandes, sobre todo la primera, y su situación marcadamente meridional, hacen que, por una parte, sea más lógico que haya un mayor número de producciones diferenciadas dada sus extensiones territoriales, y por otra, que los productos como los diversos aceites de oliva (en Andalucía hay 8 aceites de oliva con DOP) hayan podido acogerse bajo este paraguas que suponen las DOP. En el extremo opuesto se sitúan dos regiones de pequeñas dimensiones: Madrid y La Rioja. En la primera, la alta tasa de urbanización de su territorio y su testimonial tasa de actividad agraria dificultan en demasía la consecución de una DOP, mientras que en La Rioja, la orientación productivista de uno de los sectores agrarios más tecnificados del conjunto estatal ha podido ejercer como freno para lograr alguna DOP.

Las CC.AA. en las que se puede hallar un mayor número de IGP son Castilla y León y Cataluña. Este tipo de figura ampara con mucha frecuencia producciones de 
base pecuaria, básicamente cárnicas. Este hecho explica que sea Castilla la Comunidad líder en este apartado, ya que de sus diez IGP, la mitad son cárnicas. En cambio, en el caso catalán, solo dos de ellas pertenecen a esta categoría, siendo las siete restantes de origen vegetal; la mediterraneidad de la huerta catalana y por tanto, su diversidad, han sido factores decisivos para esta distribución. En el lado opuesto aparecen tres regiones tan diferentes como el archipiélago canario, la región murciana y el País Vasco. En los dos primeros casos, la menor importancia tradicional de los productos cárnicos es determinante, mientras que la situación vasca es diferente, ya que desde su gobierno autónomo se ha priorizado un modelo de etiquetaje propio al margen de los mencionados en este artículo, así que buena parte de los productos vascos de calidad se identifican con unas etiquetas o labels propias.

En lo referente a los vinos de calidad producidos en regiones determinadas, la mayor abundancia se da en Cataluña, seguida de las Islas Canarias y Castilla - La Mancha. Otra vez son las tierras catalanas las que tienen una mayor relevancia, la explicación parece residir en la acción de sus diversos actores para posicionar sus productos en los mercados mediante la vía de la calidad oficialmente reconocida, ayudados, como no, por una geografía que individualiza las producciones en muy poco espacio debido a la fragmentación y alternancia del territorio. Algo similar ocurre en Canarias, ya que su substrato y el hecho de que sea un archipiélago hacen que las producciones vitivinícolas sean plenamente diferenciadas. En el caso castellanomanchego, su extensión, su tradición, y el hecho de que se trate de tierras meseteñas con una vocación más mediterránea y cálida que las del Norte hacen que se puedan identificar hasta siete vinos diferentes con Denominación de Origen. En la situación contraria se encuentran Cantabria y Asturias, en este caso, las razones naturales son de suficiente peso para explicar la ausencia de vinos de calidad. De cualquier manera, esto no significa que no se produzcan pequeñas cantidades de vino en estos territorios, aunque solo uno de ellos, en Asturias, tiene un cierto reconocimiento, y de hecho, se incluye en la tabla en el total de productos con Denominación de Origen. Este no es un caso aislado, así que en esa última columna de la tabla se contabilizan varios vinos de la tierra en diversas regiones españolas, otra de las categorías menores de calidad. Además, también se contabilizaron los restantes productos que están reconocidos por el Ministerio de Alimentación, Agricultura y Pesca y que no entran en ninguna de las tres categorias estudiadas en este trabajo, de ahí que el número total ascienda a 242 Denominaciones de Origen. Por último, también hay que considerar que algunas de las DOP, IGP y vcprd tienen una escala pluriregional, o si se prefiere pluricomunitaria, por lo que están repartidos entre varias CC.AA. entre estos productos, podemos destacar por su representatividad, los vinos de rioja, el cava, los jamones de Huelva y Guijuelo, la carne de Ávila o el queso Idiazábal.

Todos esos productos que poseen un marchamo de calidad son los que actúan como base fundamental para el desarrollo del turismo gastronómico. De hecho, se puede afirmar que el reconocimiento a través de alguna de estas figuras consolida y 
da forma a unos recursos turísticos de carácter gastronómico que hasta hace bien poco no se habían empleado con esa intención. El reconocimiento de los productos gastronómicos los posiciona en el mercado facilitando que sobre ellos se puedan asentar productos turísticos especializados. Esta afirmación se ve corroborada al comprobar que el desarrollo del turismo gastronómico en España ha evolucionado de forma paralela al desarrollo de las distintas figuras de valorización y protección agroalimentaria.

\subsection{La creación de marcas y etiquetas de calidad en los establecimientos}

El concepto de calidad no afecta sólo a los productos alimentarios que sirven de base al desarrollo del turismo gastronómico, sino que también incumbe a los establecimientos que proporcionan los servicios ligados al mismo: nos referimos especialmente a los servicios y oferta de restauración.

El elemento más visible de todo sistema de calidad es la marca, bajo la cual se amparan un conjunto de productos turísticos y servicios que cumplen unos estándares o niveles de excelencia. La marca, prestigiosa, diferenciadora, fiable y rigurosa, proporciona ventajas para las propias empresas ya que:

- Permite la mejora constante de la misma al regirse ésta por unas normas de calidad en constante revisión.

- Favorece el posicionamiento frente a la competencia.

- Favorece la promoción y la comercialización en si.

Del mismo modo, la marca también aporta beneficios al cliente o turista ya que:

- Disminuye la incertidumbre ante la elección del establecimiento.

- Proporciona confianza y garantía de servicio.

En España, las iniciativas han sido numerosas y se han dado para diferentes ámbitos territoriales. A nivel estatal, el ICTE (Instituto para la Calidad Turística Española) ha creado la Marca de Calidad Turistica Española, bajo la cual se ampara el conjunto de los productos turísticos que cumplen con unos niveles de calidad exigidos y recogidos en las Normas de Calidad de Servicios y que aseguran estar trabajando en la mejora continua de los mismos a fin de complacer en todo momento las exigencias de los clientes.

Las Normas de Calidad de Servicio son los documentos mediante los cuales se establecen requisitos para los distintos servicios ofrecidos por entidades privadas o públicas, cuyo cumplimiento es una condición para la certificación mediante la Marca 
de Calidad Turistica Española. Las Normas Turísticas desarrolladas dentro del SCTE se sitúan en un nivel intermedio entre la ISO 9000 y el Modelo de Excelencia Empresarial (EFQM). Estas normas suponen una filosofía de gestión de calidad y de mejora continua más amplia que la ofrecida por la serie ISO 9000. Hasta el momento se han desarrollado las Normas de Calidad de 6 subsectores turísticos, entre ellas las Normas de Calidad de Restaurantes. En la actualidad, casi el 11\% de las empresas turísticas certificadas en España son restaurantes.

A nivel autonómico, uno de los ejemplos más recientes en las iniciativas tendentes a la creación de etiquetas de calidad en los establecimientos de restauración es el nombramiento de la marca de calidad Mesas de Asturias. Excelencia Gastronómica (BOPA $n^{\circ} 145$ - Lunes, 24 de junio de 2002) que responde a la necesidad de potenciar la calidad de los establecimientos de restauración del Principado de Asturias. El objetivo es facilitar al sector turístico un marco normativo apropiado para prestigiar el producto amparado y conceder a los consumidores una mayor y mejor información sobre la calidad de los establecimientos que se ofertan bajo la marca.

Un caso aparte, por su singularidad, sería también el de la creación del Proyecto de Excelencia Gastronómica ya comentado en el apartado 2 de este mismo artículo.

\section{La integración de las actividades agrarias y el turismo: estrategias para el desarrollo local}

El resultado de conectar dos sectores como son el agrario y el turístico en territorios de eminente base rural ha sido el establecimiento de estándares de calidad bidireccionales. Sin la introducción de esa consideración cualitativa, esa conjunción sería poco menos que imposible. Aún así, la pluriactividad resultante de la integración de la agricultura y el turismo se articulan de manera compleja como señala acertadamente para la pequeña región de montaña de Dévoluy (Francia) L. Rieutort (2001). Esa pluriactividad en el mundo rural es una cuestión que apareció como absolutamente necesaria para la supervivencia de éste ya en la Conferencia sobre desarrollo rural de Cork, en 1996 y que continuó estando presente en la Declaración de Salzburgo de finales de 2003. Sin duda, en los próximos años la inserción en un mismo ámbito familiar o empresarial de las actividades primarias y terciarias contribuirá a que los campos europeos cada vez tengan un carácter más multifuncional.

Con la aparición y consolidación de las modalidades turísticas alternativas, los alimentos de calidad han pasado a ocupar un lugar destacado dentro de las preferencias tanto de los restauradores locales como de los turistas. De este modo, se puede 
afirmar que los productos de calidad (estén o no amparados por un distintivo a tal efecto) han servido como base al desarrollo del turismo gastronómico. Aunque, el que un producto posea algún tipo de denominación de origen se convierte en un elemento fundamental en la mejora de su competitividad. Por tanto, los dos sectores, el agrario y el turístico, quedan indisociablemente unidos en un contexto rural que comienza a valorar positivamente aquello que hasta hace bien poco era considerado como algo negativo.

Este tipo de asociación está directamente promovida por la política de desarrollo rural que descle finales de la década de los noventa se plantea en la UE. Estaría incluida dentro de lo que M. Jouen (2001) denomina la visión extrovertida del desarrollo rural, o lo que es lo mismo, aquella que apuesta fundamentalmente por la importación de la modernidad, presentándose como programas de desarrollo económico para las actividades terciarias (turismo, patrimonio cultural) o primarias (agricultura y pesca), mejora de la calidad y comercialización de las producciones agrarias y de origen artesanal. Dichas actuaciones, están inscritas dentro de las previstas desde la iniciativa comunitaria LEADER; esta iniciativa junto con el programa PRODER son dos de los ejes en los que se articula la moderna política de desarrollo rural europea. J. Esparcia (2001) le atribuye a estos programas una importancia relativa, ya que si bien son los que demuestran un enfoque más territorial, no son cuantitativamente los más importantes. El monto económico de los enfoques sectoriales y de las medidas de acompañamiento es mucho mayor que el que se manejan desde los dos programas mencionados.

Las actuaciones en materia de desarrollo rural inscritas en el marco de la iniciativa LEADER arrancan en el año 1991, y durante sus tres primeros años de vida se centran, precisamente en el desarrollo del turismo rural y en la revalorización de los productos agrarios, entre otras cuestiones de importancia como la potenciación de la artesanía y la formación profesional. Enlazando con esta primera iniciativa, aparece lo que hasta 1999 se denominó LEADER II y PRODER, ambos muy similares entre sí (Esparcia, 2003); en este segundo período, las acciones más numerosas se dieron en los ámbitos de las actividades turísticas, la promoción de pequeñas empresas industriales y de servicios, y el desarrollo y mejora de las empresas de elaboración y comercialización de recursos agrarios; solo estos tres epígrafes concentraron las tres cuartas partes de la inversión total (Alario Trigueros, 2001). A partir de 1999 ha entrado en funcionamiento la tercera fase de la iniciativa, que lleva por nombre LEADER +, que tiene tres objetivos básicos: a) la valorización del patrimonio tanto natural como cultural; b) la mejora de las economías rurales mediante la creación de empleos; c) la mejora de la capacidad organizativa de las comunidades rurales. Además, también el programa PRODER tiene su continuación con una segunda parte que llega, como en el caso de LEADER, hasta 2006. 
En la actualidad, el concepto de desarrollo rural ha de incluir una serie de caracteres que hasta hace poco tiempo ni siquiera se antojaban como necesarios de manera remota. Así, hoy en día, aspectos como el respeto medioambiental, la equidad social, el mantenimiento y recuperación cultural, y la interconectividad y multifuncionalidad laboral y económica se manifiestan como absolutamente necesarios para la supervivencia del mundo rural. Los sectores económicos no pueden permanecer aislados sino que deben complementarse, retroalimentándose y promocionándose respectivamente. Esta es la base sobre la que se sustenta la relación entre el turismo gastronómico y las producciones agrarias de calidad. Así, es cada vez más frecuente ver en la carta de restaurantes del medio rural español informaciones que complementan la descripción del plato en concreto mediante la indicación de la figura de protección bajo la que están amparados (p. ej. chuletillas de cordero DOP Ternasco de Aragón, alcachofas de Benicarló -IGP- con almejas, etc.). Esta estrategia de diferenciación ha conllevado un aumento del número de ferias comerciales, fiestas populares, revistas especializadas, libros de cocina y folletos turísticos dedicados a los productos regionales, demostrándose de este modo el creciente interés en toda Europa por este tema.

En las diferentes fases de la iniciativa comunitaria LEADER y de los programas PRODER la valorización de los productos agrarios ocupan un papel destacado. De hecho, existen numerosos ejemplos en el conjunto español de este tipo de actuaciones; así, hace más de diez años que en la comarca de La Serena (Extremadura) se han valorizado los productos como el queso de oveja y la carne de cordero; en varias comarcas andaluzas de montaña (Sierra Morena, Serranías de Ronda y Grazalema, etc.) son los recursos cinegéticos mayores como el jabalí, el corzo o el ciervo los que se están consolidando como base de una gastronomía diferenciada y atrayente para los turistas; también en tierras andaluzas, en concreto en la Sierra de Aracena, es el jamón amparado bajo la DOP Jamón de Huelva, junto con la micología, el eje alrededor del cual se articula buena parte de la promoción turística de esta área ${ }^{6}$; en Aragón, en su zona prepirenaica, la pequeña localidad de Luesia es conocida por sus judías, y gracias al apoyo del Grupo de Acción Local (GAL) interautonómico del Prepirineo Navarro-Aragonés, se celebra en ella una feria que tiene como protagonista este producto, donde conviven la venta del producto, la gastronomía y las conferencias y talleres de corte técnico, sirviendo todo ello para atraer visitantes de las dos comunidades e incluso de fuera de esas dos regiones; en la isla de La Palma (Islas Canarias) hace cinco años que se creó una asociación de productores agroalimentarios de calidad; desde ella, además de realizarse acciones en pos de la mejora productiva, también se ha creado un paquete de productos que bajo el nombre comercial de Tesoro Gastronómico, ofrece los productos típicos artesanales ${ }^{7}$ de la isla a los turistas; la demanda turística también jugó un papel fundamental en el establecimiento y creación de una marca de calidad regional que ampara la carne de vacuno que se produce en el ámbito de actuación del LEADER Pinares-El Valle, a caballo entre las provincias de Soria y Burgos. 
El turismo gastronómico no es sólo un fin para la consecución del desarrollo local sino que además se configura como una herramienta básica para la promoción y distribución de los productos agroalimentarios locales cuya comercialización debe procurar también el desarrollo local. Esto es lógico si pensamos que una de las características que definen a este tipo de producciones es su carácter local y su vinculación con la pequeña empresa, esta última con dificultades para publicitar y vender sus productos. Es por ello que: a) La creación de infraestructura turística básica (restaurantes, casas rurales, hoteles...) con el fin de que los productores de alimentos utilicen los establecimientos para dar a conocer sus productos; b) La creación de museos, vinacotecas, centros de interpretación, asî como organización de eventos y mercados que permitan la aproximación y conocimiento de los activos del territorio; y c) La vinculación de la promoción turística de la comarca con la existencia de productos agroalimentarios de calidad se configura como una estrategia básica para la promoción. De hecho, el turista y el excursionista de paso se configuran como la salvación para las pequeñas empresas, no sólo al convertirse en vectores para la difusión, sino también al facilitar la autocomercialización, es decir, la venta directa por parte de los propios operadores inscritos en los registros de los Consejos Reguladores. Existe la esperanza de que esta estrategia de comercialización consolide un mercado potencial de consumidores de nivel medio-alto. De hecho, el desarrollo de un turismo temático conlleva la especialización en un público objetivo (el interesado en las cuestiones gastronómicas) que, casualmente, coincide con el mercado real o potencial de esos alimentos de calidad.

\section{Consideraciones finales}

En los últimos años es más que evidente el surgimiento de nuevas modalidades turísticas que han ocupado nichos territoriales y de mercado que hasta este momento habían pasado desapercibidos por parte de los agentes comercializadores y por ende por los consumidores. Al mismo tiempo, los campos españoles comenzaron una carrera hacia la valorización de sus productos al amparo de figuras plenamente legisladas que establecen una relación directa entre la calidad y el origen geográfico de los mismos. El resultado de este entramado ha sido la retroalimentación entre los dos sectores productivos; el primario y el terciario se dan la mano para posicionarse de manera firme en un mercado en el que la tradición juega un papel muy importante. Los productos agroalimentarios se configuran, en este contexto, como recursos turísticos gastronómicos que posibilitan el desarrollo del turismo y, a la vez, el turismo se convierte en herramienta para la promoción y comercialización de los productos agroalimentarios de calidad. Los nexos que se han establecido entre las producciones agropecuarias de calidad y esas nuevas modalidades turísticas han dado como resultado la aparición de una gastronomía que recupera modos tradicionales y al mismo 
tiempo innova en nuevas propuestas y que tiene como base esos productos de calidad. Los dos sujetos estudiados en este artículo, las producciones y el turismo de calidad, actúan por tanto, como dos herramientas que a través de la gastronomía o de la venta por otros canales contribuyen al desarrollo local de numerosas regiones y localidades que quieren jugar un papel dentro de unas pautas de consumo cada vez más vinculadas a lo urbano.

En definitiva, los productos agroalimentarios de calidad y todo lo que gira a su alrededor son el punto de partida de algunos de los proyectos de desarrollo rural que se han realizado en los últimos años en buena parte de las áreas consideradas como marginales desde la perspectiva económica y que están sirviendo para dinamizar esos territorios, no solo desde la ya citada perspectiva económica, ya que desde el punto de vista social e incluso en ocasiones desde el ambiental esas regiones se están viendo potenciadas.

\section{Agradecimientos}

Este artículo ha sido realizado en el marco de un proyecto de investigación sobre Desequilibrios territoriales, mercados de trabajo y áreas marginales en Cataluña, financiado por la CYCIT (proyecto BSO2001-3095) y con un Ajut de Suport a la Recerca dels Gmips Consolidats del II Pla de Recerca de la Generalitat de Catalunya (Grup de Recerca d'Anàlisi Territorial i Desenvolupament Regional, 2001SGR-00016), ambos dirigidos por la Dra. Roser Majoral.

\section{Notas}

1 En 1981 se publicó el memorándum Guidelines for the European Agriculture donde ya aparecía la preocupación por los aspectos sociales relacionados con la pérdida de empleo agrario. Otro de los hechos fundamentales que se produjo a mediados de esta década, en 1984, es el establecimiento de las tasas del sector lácteo para intentar hacer frente al problema de los excedentes. También se debe destacar que en 1985 los temas medioambientales aparecen por primera vez como protagonistas de la normativa agraria comunitaria gracias al Reglamento $n^{\circ} 797 / 85$ de 12 de marzo relativo a la mejora de la eficacia de las estructuras agrarias.

2 Según la Federación Española de Hostelería, en el año 2002, existían en nuestro país 254.802 bares, 54.520 restaurantes y 12.696 cafeterías, habiéndose duplicado prácticamente las cifras de todos estos establecimientos respecto al año 1985. Del mismo modo, comparativamente, el subsector de la restauración muestra un ritmo de expansión mucho más acelerado que el resto de subsectores turísticos: en el período 1975-2000, el subsector del alojamiento sólo incrementó el número de plazas en algo más del $50 \%$ mientras que el número de plazas en restaurantes para el mismo período evolucionó triplicándose (AECIT, 2002; 2003). 
3 Con anterioridad, en 1991, se reguló mediante el Reglamento (CEE) 2092/91 la producción agraria ecológica, otro de los hitos fundamentales en lo que a valorización de las producciones agrarias se refiere.

4 Hay que reseñar que la definición de esta figura en ningún momento aparece del todo clara a lo largo de las 99 páginas de que consta el reglamento, produciéndose una cierta indefinición en las escalas utilizables (una denominación para un vino de calidad puede ir descle la escala inframunicipal que supone un predio a abarcar varias Comunidades Autónomas).

5 Entre esos países los más significativos por su beligerancia en este aspecto son Canadá, Australia, Nueva Zelanda y Argentina. Desde ellos se acusa de imperialismo cultural a la UE, aunque la mayoría de estos productos no tengan ni por asomo las mismas cualidades que los originales europeos.

6 A este respecto, para comienzos del verano 2004 está prevista la inauguración del Museo del Jamón en la localidad de Aracena, en él los visitantes podrán conocer el ecosistema de la dehesa, las poblaciones de la sierra, los caracteres esenciales del cerdo ibérico, su matanza y la curación y cuiclado del jamón, además de lógicamente poder degustar el producto.

7 Dentro de ese conjunto de productos típicos de La Palma figuran cigarros puros artesanales, miel, licores de frutas, mojo rojo, dulces elaborados con almendras y vino Malvasía.

\section{Bibliografía}

AECIT (2002): La actividad turistica española en 2001 (Edición 2002). Pub. AECIT (Asociación Española de Expertos Científicos en Turismo), Castellón de la Plana.

AECIT (2003): La actividad turistica española en 2002 (Edición 2003). Pub. AECIT (Asociación Española de Expertos Científicos en Turismo), Castellón de la Plana.

AFRAT (2001): Des réseaux pour valoriser les cuisines du terroir, La Gazette Officielle du Tourisme, 1601, p. 4-6.

Alario Trigueros, M. (2001): Las políticas de planificación y de desarrollo de los espacios rurales, en García Pascual, F. (coord.): El mundo mural en la era de la globalización: incertidumbres y potencialidades, p. 213-265.

Antón Clavé, S. (2000): Turismo, territorio y cultura, en Morales Matos, G. (ed.), Turismo y Ciudad, Las Palmas de Gran
Canaria, Ayuntamiento de Las Palmas de Gran Canaria y A.G.E., p. 29-41.

Armesto López, X.A. (2003): Agricultura ecológica y postproductivismo: un estudio comparado de Cataluña y Galicia. Barcelona, Tesis Doctoral inédita, UB.

Au, N. y Law, R. (2002): Categorical classification of tourism dining. Annals of Tourism Research, 29 (3), p. 819-833.

Chon, K.S. (1990): The role of destination image in tourism: a review and discussion. Revue du Tourisme, 2, p. 2-9.

Esparcia Pérez, J. (2001): Las políticas de desarrollo rural: evaluación de resultados y debate en torno a sus orientaciones futuras, en García Pascual, F. (coord.): El mundo mural en la era de la globalización: incertidumbres y potencialidades, p. 267-309.

Esparcia Pérez, J. (2003): Valoración y balance de PRODER. Versión Original. 
LEADER. Revista de Desarrollo Rural, 22, p. 16-19.

Gallarza, M., Gil, I. y Calderón, H. (2002): Destination image: towards a conceptual framework. Annals of Tourism Research, Vol. 29, 1, p. 56-78.

Grande Ibarra, J. (2001): Análisis de la oferta de turismo cultural en España, Estudios Turisticos, 150 , p. 15-40.

Grigg, D. (1999): Food consumption in the Mediterranean Region. Tijdscbrift voor Economiscbe en Sociale Geographie, 90 (4), p. 391-409.

Haukeland, I. y Jacobsen, K. (2001): Gastronomy in the periphery. Food and cuisine as tourism attractions on the top of Europe. $10^{b}$ Nordic Tourism Research Conference, Vasa, Finland (18-20 October 2001).

Hervieu, B. (1996): Los campos del futuro. Madrid, Serie Estudios $n^{\circ} 118$, Ministerio de Agricultura, Pesca y Alimentación, Secretaría General Técnica.

Hudman, L. (1984): The travellers' perception of the role of food and eating in the tourist industry. En The impact of catering and cuisine upon tourism, Suisse, AIEST, p. 95-105.

Hughes, G. (1995): Authenticity in tourism. Annals of Tourism Research, 22 (4), p. 781-803.

Ilbery, B. \& Kneafsey, M. (2000): Producer constructions of quality in regional speciality food production: a case study from south west England. Journal of Rural Studies, $16 / 2$, p. $217-230$.

Ilbery, B., Kneafsey, M., Bowler, I. y Clark, G. (2000): Consumers perspectives on qua- lity products and services from lagging rural regions in the European Union, en Molinero, F. et al. (coords.): Ir Simposio Anglo-Español de Geografía Rural, Valladolid, Universidad de Valladolid, edición CD-ROM.

Ivars, J.A. (2000): Turismos y Espacios Rurales: Conceptos, filosofias y realidades. Investigaciones Geográficas, 23, p. 59-88.

Jouen, M. (2001): La Europa rural al principio del tercer milenio. La palabra clave: diversidad. LEADER Magazine, 25, p. 5-12.

Leslie, D. y Reimer, S. (1999): Spatializing commodity chains. Progress in Human Geography, 23/3, p. 401-420.

López Palomeque, F. (1999): Política turística y territorio en el escenario de cambio turístico. Boletín de la A.G.E., 28, p. 23-38.

Mckercher, B. (2002): Towards a classification of cultural tourists. International joumal of Tourism Research, 4, p. 29-38.

Montero, I., Gutiérrez, D. y Díaz, R. (2001): La cultura como componente de la oferta de los destinos turísticos maduros. Estudios Turisticos, 150, p. 41-55.

Moulin, C. (2000): Gastronomy and tourism: Must every tourist wish be our command? Revue du Tourisme, 1, p.19-24.

Rieutort, L. (2001): Tourisme, agriculture et nouvelles activités en espace rural : l'autre légende de Dévoluy, en Berger, A. (dir): Dynamique rurale, environnement et stratégies spatiales, p. 345-356.

Ritzer, G. (1998): The McDonaldization Thesis. Londres, Ed. Sage. 
Rubio, P. (1999): El postproductivismo en los espacios rurales, en Galdós, R. y Ruiz, E. (coords.), Postproductivismo y Medio Ambiente, perspectivas geográficas sobre el espacio miral. Vitoria-Gasteiz, Informes técnicos $\mathrm{n}^{\circ}$ 82, Dpto. de Agricultura $\mathrm{y}$ Pesca, Gobierno Vasco.

Sage, C. (2003): Social embededness and relations of regard: alternative "good food" networks in south-west Ireland. Journal of Rural Studies, 19, p. 47-60.

Telfer, D. (2001): Strategic alliances along the Niagara Wine Route. Tourism Management, 22, p. 21-30.

Telfer, D. y Wall, G. (1996): Linkages between tourism and food production. Annals of Tourism Research, 23 (3), p. 635-653.
UNESCO (1972): Convention concerning the protection of the world cultural and natural beritage. Paris.

UNESCO (1998): Conferencia intergubernamental sobre politicas culturales para el desarrollo. Estocolmo.

Verhaegen, I. \& Van Huylenbroeck, G. (2001): Costs and benefits for farmers participating in innovative marketing channels for quality food products. Journal of Rural Studies, 17/4, p. 443-456.

Waller, J. and Lea, S. (1998): Seeking the real Spain?. Authenticity in Motivation. Annals of Tourism Research, 25 (4), p. 110-129.

Winter, M. (2003): Embeddedness, the new food economy and defensive localism. Journal of Rural Studies, 19, p. 23-32. 